\title{
The rare cervical MDR-tuberculosis lymphadenitis infection in an infant from Mashhad, Iran; a case report and literature review
}

\author{
Masoud Keikha ${ }^{1}$, Fatemeh Askarizadeh ${ }^{1}$, Mohammad Saeed Sasan ${ }^{2}$, Hossein Joghatayee ${ }^{2}$, \\ and Saman Soleimanpour ${ }^{3}$ \\ ${ }^{1}$ Mashhad University of Medical Sciences Emam Reza Hospital \\ ${ }^{2}$ Mashhad University of Medical Sciences Dr Shariati Hospital \\ ${ }^{3}$ Mashhad University of Medical Sciences Bu Ali Avicenna Research Institute
}

August 3, 2020

\begin{abstract}
Herein we report the first MDR-TB case of Cervical tuberculosis lymphadenitis from patients which was born form susceptible TB cured mother in Mashhad, Iran.
\end{abstract}

\section{Key Clinical Message}

- Multi drug-resistant cervical tubercular lymphadenitis is uncommon, but there are cases in TB endemic region.

- Clinical diagnosis of multi drug-resistant cervical tubercular lymphadenitis is difficult and may be missed.

- The fluoroquinolones (moxifloxacin) is appropriate prophylactic for family members of such cases.

\section{Introduction}

Tuberculosis is one of the most important infectious diseases that is considered among the top ten causes of mortality worldwide. According to the reports by the World Health Organization (WHO), about 10 million new cases of tuberculosis were diagnosed in 2018, of which 1 million were children; 1.6 million related mortalities were also reported in the same year. Currently, it has been estimated that one-fourth of the world's population is suffering from the latent TB infection (LTBI), 5-10\% of whom will encounter active TB in future [1-2]. The statistics regarding Mycobacterium tuberculosis (Mtb) drug resistance is also very worrying; 558,000 rifampicin-resistant cases were specified in that year, $82 \%$ of whom were multi-drug resistant TB (MDR-TB). According to this report, Iran is also included among the endemic tuberculosis countries [2-3]. The rate of incidence of tuberculosis in Iran is about 22 per 100,000 and the mortality rate is 3.5 per 100,000. According to the reports, the rate of rifampicin and MDR-TB resistance has been reported in Iran at about $1.3-5 \%$ [4-5].

While most of the clinical manifestations of tuberculosis are in the pulmonary basis, however, $M t b$ is capable of affecting all the organs, which is termed extra-pulmonary TB (EPTB). In this respect, cervical tuberculous lymphadenitis is the most common form of EPTB, accounting for 25-30\% of the cases [6]. The incidence rate for the EPTB in Iran has also been reported to be approximately 2.5 cases per 100,000 people per year, which are mainly children [7]. Cervical tuberculosis lymphadenitis (CTL) typically involves the lymph nodes of the jugular, posterior triangle, and supraclavicular region, and the observed clinical manifestations of cervical tuberculosis lymphadenitis include fever, weight loss, rarely coughing, night sweat, chills, malaise, suppurative lymphadenitis, granulomatous inflammation, neck mass $(1-3 \mathrm{~cm})$, fistula formation and 
caseous necrosis. However, cervical tuberculosis lymphadenitis is confused with the diseases such as malignancy, fungal infection, tularaemia, actinomycosis, sarcoidosis, and non-tuberculosis mycobacteria (NTM) lymphadenitis [8-11]. Studies have shown that the accuracy and sensitivity of the diagnosing methods for cervical tuberculosis lymphadenitis, such as Ziehl-Neelson (ZN) staining, microbiological culture and PCR, have little diagnostic value and are not reliable, particularly in the medication-resistant cases. The physician may be mistaken for the misdiagnosis due to failing to respond to the anti-tuberculosis treatment [12-15].

Based on the WHO recommendations, category III tuberculosis has been used since the beginning of 1997 to treat the cervical tuberculosis lymphadenitis cases [9]. The development of drug resistance TB (particularly in TB endemic regions) has led to poor treatment outcomes in recent years [16]. Thus, there is a new alternative treatment beside the anti-TB therapy that has been introduced for adults, which is surgery. These invasive techniques also have their own risks and need to be modified and standardized according to the cervical tuberculosis lymphadenitis guidelines [9,16-17]. According to review of the literature, there are numerous reports about cervical tuberculosis lymphadenitis in children [18-19]. However, there are only 5 reports regarding MDR-CTL infection throughout the worldwide. All of these cases had between 19-35 years of age, who recovered by administration of the second-line anti-TB agents [20-24]. We were informed about the first case of congenital cervical tuberculosis lymphadenitis in a 2-months old infant, who was infected by MDR-Beijing $M t b$ strain. The aim of the present study was to describe an unusual report of cervical MDR-tuberculosis lymphadenitis in a 2-months age infant from Mashhad, Iran.

\section{Case presentation}

In August 2018, a 32-year-old pregnant mother at her 34 weeks of gestation was referred to Dr. Shariati Hospital in Mashhad, Iran, due to weight loss, fever, persistent, coughing and dyspnea. She was diagnosed by sputum smear and culture examination to have pulmonary tuberculosis. The patient was treated for category I tuberculosis, and her symptoms became gradually negative in accordance with the sputum smear results. However, she delivered about one month later at 41 weeks gestation. Despite receiving prophylactic TB therapy (isoniazid), 55 days after his birth, the child (boy infant) was affected to fever $\left(38.3^{\circ} \mathrm{C}\right)$, anorexia and a painful swollen lesion in the neck area, and was taken to Dr. Shariati Hospital in Mashhad. His parents had no consent for lumbar puncture. Therefore, since the mother had tuberculosis during pregnancy, the baby was also diagnosed as a "TB Case" and treated with isoniazid and rifampicin. Although the neck mass biopsy results showed evidence of inflammation but they were negative for the acid-fast bacilli. The child's cervical swelling did not improve after about 4 months of anti-tuberculosis therapy, and had a pale colored discharge.

The purified protein derivative (PPD) test indicated about $3 \mathrm{~mm}$, when he was 7 months old. Moreover, the patient was also negative for the HIV, HBV and HCV tests. The patient had no evidence of organomegaly. CBC results included WBC: $24,000 / \mathrm{mm}^{3}$, RBC: $5200 / \mu \mathrm{m}, \mathrm{Hb}: 11.5$ g.dl, HCT: $37.4 \%$ and Platelets: $535,000 / \mathrm{mm}^{3}$. The serum levels of IgG, IgM and IgA were 1500, 122 and $104 \mathrm{mg} . \mathrm{dl}$, respectively. The parents of his patient were not consent for lumbar puncture of baby and disseminated TB was ignored based on the radiological findings and negative culture of blood for $M t b$. The sonography analyses of the internal organs such as liver, bile duct, pancreas, kidney and bladder were normal and no evidence of pleural effusion; Chest X-ray (CXR) images of the patient's lung showed normal results (Figure 1).

The patient underwent FNA (Fine Needle Aspiration) and the patient's biopsy specimen was sent to Tuberculosis Reference Laboratory in Mashhad for cytopathology and culture studies. Based on reported results from the neck mass biopsy, numerous evidences of multiple necrotizing granuloma with caseous lesion were observed; Ziehl-Neelsen staining results confirmed the existence of acid-fast bacilli. The results of the "QuantiFERON assay" also identified the presence of an immunological response to $M t b$ infection.

The standard proportional method analysis was carried out according to the Clinical and Laboratory Standards Institute on Lowenstein-Jensen medium and resistance was observed to isoniazid, rifampicina and ethambutol. According to the GeneXpert MTB-RIF assay, the Mtb isolate was resistant to rifampicin. In addition, based on the drug molecular susceptibility test (DST) results performed by DNA sequencing, the 
considered isolate was resistant to rifampicin, isoniazid and ethambutol and was introduced as an MDR-TB case. Due to the importance of identifying source of infection, the $M t b$ isolates of the studied infant and her mother underwent genetic fingerprinting subjected to IS6110-RFLP and Spoligotyping. It was found that the infant isolate was from the Beijing lineage, whereas that of her mother's was from the mixed infection with Beijing and Delhi/CAS lineages. Confirmation of IS6110-RFLP results by Spoligotyping suggested that the mother had multiple strains as the consequence of recent transmission. It has been more probable that the patient was infected with $M t b$ as congenitally route. However, the patient was treated with a combination therapy including surgical drainage and antibiotic therapy by ethionamide, moxifloxacin, amikacin and linezolid. Other members of the patient's family also received prophylactic MDR-TB (with moxifloxacin). After one year and regular follow-up of the patient, it was found that the patient had fully recovered and no signs of reactivation were observed in the patient, his mother, and their other family members.

\section{Discussion}

Drug resistant TB has been one of the most important public health challenges that we have faced since the early 1990s [25]. Due to the fact that Iran is TB endemic, as well as its proximity to TB high burden countries, several cases of DR-TB (Drug resistance TB) are reported annually in this country [26-28]. Based on the existing information for the new and previously reported treated cases, the MDR-TB prevalence in Iran is reported to be $2.48 \%$ and $5 \%$, respectively [22]. Reports on DR-TB indicate its annual increase due to the spread of MDR-TB strains worldwide, and in particular in the Middle-East region [29]. One of the most important DR-TB problems is the high mortality rate in DR-TB cases and the increase of primary drugresistant TB cases [30]. Currently, 5 different types of DR-TB have been reported, including mono-resistance TB, poly resistance TB, multi-drug resistance TB (MDR-TB), extensively-drug resistance TB (XDR-TB) and totally-drug resistance TB (TDR-TB), for which monitoring, controlling and preventing the prevalence of MDR-TB, XDR-TB and TDR-TB are of great importance [31].

The present study was a rare report about cervical lymphadenitis caused by primary MDR-TB strain in an infant that was born from TB patient mother. The infant was considered as MDR-TB case, while his mother was susceptible to the first line anti-TB drugs. Fingerprinting of infant and mother isolates showed that the mother was infected with multiple strains, whereas the child was infected with the strains of the Beijing genotype family. Therefore, it is more probable that the infant was infected from mother as the congenital route. The MDR-TB strain for the present case belonged to Beijing genotype family, which was confirmed previously in the published Iranian reports. In a systematic review and meta-analysis on the Iranian MDR-TB cases, Tarashi et al. (2017) found that the distribution of Beijing genotype family is predominant genotypes among the MDR-TB Iranian cases [32-33].

Congenital tuberculosis is occurred as a result of umbilical vein into utero, perinatal route via ingestion or aspiration of infected amniotic fluid or direct contact with the infected parental genital lesions. Infected placenta is mainly the route of congenital TB in neonates that can transmit $M t b$ strains to the fetal liver or lungs [34-35]. The primary TB infection symptoms can be presented as pulmonary complication disseminated to the internal organs, cutaneous or central nervous system [36]. According to review of the literature, Cantwell et al. (1994) found that the age range presenting the congenital TB symptoms is between 24-84 days. We observed the sign of TB in our case at 55 days after his birth [37]. We also found that the mother was infected by multiple $M t b$ strains. Thus, the possibility of confronting with a congenital TB case is higher, although the boy infant had not responded to the first-line anti tuberculosis drugs, as opposed to his mother. Infants are more susceptible to tuberculosis due to their lack of sufficient immunity system. There are some reports about the cervical tuberculosis lymphadenitis in children below 1-year of age. We report the first case of MDR-TB cervical lymphadenitis infection, which could have occurred due to spontaneous mutations in response to the inadequate anti-TB therapy as well as primary resistance to the Beijing strains in Iran [38-40].

Cervical tuberculosis lymphadenitis is one of the most prevalent forms of extra-pulmonary TB that often occurs in immunocompromised cases [23]. Although $M t b$ often affects the lungs, in the immune-compromised cases, particularly children and HIV-infected people, TB bacilli are spread through through the lymphatic 
system due to the lack of an efficient and effective TB bacilli immune system, often occurring in the form of Cervical tuberculosis lymphadenitis [23, 41-42]. Despite the fact that the CTL cases caused by DR-TB are very limited, but the epidemiologic importance and diagnostic difficulties of managing and treatment of these cases are quite challenging because of the lack of specific guidelines for the treatment, especially in the immunocompromised patients, who do not usually have granulomatous inflammation due to immune dysfunction. Moreover, PPD results in these patients are negative due to a weakened immune system [23, 42]. Based on the available evidence, lung CXR is usually normal in cervical tuberculosis lymphadenitis patients, with merely showing the abnormalities in $24-46 \%$ of these patients [43]. So far, limited cases of cervical tuberculosis lymphadenitis caused by DR-TB strains have been reported, most of whom are in the TB endemic countries (Table 1).

According to the review of the literature, lymphadenopathy is the most prevalent form of TB in the endemic TB countries, whereas infection with DR-TB strains is rare in these countries [23], although regarding the increased prevalence of DR-TB, increasing the number of these cases is not unexpected in the coming years $[22,42]$. The most common clinical manifestations and symptoms of cervical tuberculosis lymphadenitis include single or multiple painless lumps, lymphadenopathy, fistula formation (in some cases), weakness, low grade of fever, coughing and pulmonary hilar lesion (if being involved and in case of primary lung infection) [43-44]. Cervical tuberculosis lymphadenitis may lead to misdiagnosis in cases with negative PPD results, lack of evidence of lung involvement, absence of granuloma formation in some cases, and coinfection with HIV or immune-disorder [45]. However, no relapse in the untreated patients is observed and mortality rate is reported to be low in the DR-TB cervical lymphadenitis patients, although it is not being fatal if promptly diagnosed and treated (Table 1).

Fine needle aspiration is a precise and reliable tool for cervical tuberculosis lymphadenitis detection, that based on available sources, their sensitivity and specificity are about $88 \%$ and $96 \%$, respectively, based on available sources [46]. However, according to Deveci et al. (2016), the sensitivity of acid-fast staining and culture methods for cervical tuberculosis lymphadenitis detection are estimated to be about $46-78 \%$ and $10-69 \%$, respectively [47]. Culture also takes about 6-8 weeks due to the slow growth nature of $M t b$ and is not quite appropriate. However, the molecular methods, particularly IS6110 -PCR, are able to detect the cervical tuberculosis lymphadenitis cases in a very short time with acceptable accuracy [45-47]. According to the previous studies, the positive results of PCR obtained by FNA on the cervical tuberculosis lymphadenitis samples were high, being $71.4 \%, 76.4 \%$ and $92.1 \%$, respectively in the three fulfilled studies [48-49]. Drugresistant-TB (DR-TB) CTL treatment had a low mortality rate despite the lack of standard guidelines, and it seems that combination therapy, including sinus drainage and anti-tuberculosis drugs based on drug susceptibility testing, to have satisfactory results and usually relapse in these cases (Table 1).

\section{Conclusion}

A rare case of MDR-TB cervical lymphadenitis in a 2-month male infant was reported from the mother with pulmonary TB. According to results of $M t b$ strains genotyping, it was determined that his mother was infected by the multiple $M t b$ strains and possibly the infant boy was infected with regards to the congenital-TB route rather than the environment. Congenital TB symptoms were various in the infants and it could be converted to TB-disseminated infection. There was no evidence of disseminated infection for the present case using radiological findings, laboratory indexes and bacterial culture testing. Surprisingly, we confronted with an MDR-TB infant case delivered from a drug-susceptible pulmonary-TB mother case, which is quite uncommon. The physician decision was initiated with the first-line anti-tuberculosis therapy without the DST (Drill Stem Test) result due to time-consuming aspect of the $M t b$ culture and this peculiar emergency case. However, we performed DST by the molecular techniques and identified that the infant was an MDR-TB case. There are several points to be considered in this case, which including (i) Iran is TB endemic region and it is necessary to introduce new local TB control programs such as performing DST and anti-tuberculosis regimen should be recommended based on the DST pattern in such a region; (ii) Clinical manifestations of cervical tuberculosis lymphadenitis were not very unique and could be confused with other infections, particularly in the absence of the response to Category I anti-TB regimen treatment due to the 
DR-TB infection, as in the present case; (iii) The fine needle aspiration (FNA) technique with the molecular methods can diagnose the cervical tuberculosis lymphadenitis cases with great accuracy; (iv) Although the DR-TB strains should be diagnosed quickly, the cervical tuberculosis lymphadenitis infections caused by these strains are not usually fatal, in case they are properly administered and given antibiotic-based drug susceptibility testing; (v) Fluoroquinolone is an appropriate therapeutic agent for the prophylactic aspect of contacts with the MDR-TB cases.

\section{Declarations}

\section{Ethics approval and consent to participate}

The ethical approval for performing this study was obtained from the Ethics Committee of the Mashhad University of Medical Sciences and written informed consent was obtained from parent's patient.

\section{Consent to publish}

Consent of publish form were obtained from his parent's patient.

\section{Availability of data and materials}

Not applicable.

\section{Competing interests}

The authors declare that they have no competing interests.

\section{Funding}

Not applicable.

\section{Authors' Contributions}

Writing original draft: MK and FA; Writing - review \& editing: MK and SS; Investigation and data analysis: MSS and HJ; Supervision: MK and SS.

\section{Acknowledgements}

We thank from Mashhad University of Medical Sciences and Dr. Sheikh hospital staff for supporting us to perform the current study.

\section{References}

1. Keikha M, Esfahani BN. The relationship between tuberculosis and lung cancer. Adv Biomed Res . 2018;7:58.

2. World Health Organization. Global tuberculosis report 2018. World Health Organization; 2018.

3. Tacconelli E, Carrara E, Savoldi A, Harbarth S, Mendelson M, Monnet DL, Pulcini C, Kahlmeter G, Kluytmans J, Carmeli Y, Ouellette M, et al. Discovery, research, and development of new antibiotics: the WHO priority list of antibiotic-resistant bacteria and tuberculosis. Lancet Infect Dis . 2018;18(3):318-27.

4. Zaniani FR, Moghim S, Mirhendi H, Safaei HG, Fazeli H, Salehi M, Esfahani BN, et al. Genetic Lineages of Mycobacterium tuberculosis Isolates in Isfahan, Iran. Curr Microbiol . 2017;74(1):14-21.

5. Bialvaei AZ, Asgharzadeh M, Aghazadeh M, Nourazarian M, Kafil HS. Challenges of Tuberculosis in Iran. Jundishapur J Microbiol . 2017;10(3).

6. Xu JJ, Peer S, Papsin BC, Kitai I, Propst EJ. Tuberculous lymphadenitis of the head and neck in Canadian children: Experience from a low-burden region. Int J Pediatr Otorhinolaryngol . 2016; 91:11-4.

7. Lotfian F, Bolursaz MR, Tabarsi P, Velayati A. Comparison Between Pulmonary and Extrapulmonary Tuberculosis in Adolescents. Arch Pediatr Infect Dis . 2017;5(3).

8. Karabay OĞ, Kilic S, Gurcan S, Pelitli T, Karadenizli A, Bozkurt H, Bostanci S; et al. Cervical lymphadenitis: tuberculosis or tularaemia? Clin Microbiol Infect . 2013;19(2):E113-7. 
9. Oishi M, Okamoto S, Teranishi Y, Yokota C, Takano S, Iguchi H, et al. Clinical study of extrapulmonary head and neck tuberculosis: a single-institute 10-year experience. Int Arch Otorhinolaryngol . $2016 ; 20(1): 30-3$.

10. Ukekwe FI, Olusina DB, Banjo AA, Akinde OR, Nzegwu MA, Okafor OC, Ocheni S, et al. Tuberculous Lymphadenitis in South-Eastern Nigeria; a 15 Years Histopathologic Review (2000-2014). Ann Med Health Sci Res . 2016;6(1):44-9.

11. Gautam H, Agrawal SK, Verma SK, Singh UB. Cervical tuberculous lymphadenitis: clinical profile and diagnostic modalities. Int $J$ Mycobacteriol . 2018;7(3):212.

12. Tachibana T, Orita Y, Fujisawa M, Nakada M, Ogawara Y, Matsuyama Y, Abe I, Sato Y, Uesaka K, Nishizaki K, et al. Factors that make it difficult to diagnose cervical tuberculous lymphadenitis. $J$ Infect Chemother . 2013;19(6):1015-20.

13. Moualed D, Robinson M, Qureishi A, Gurr P. Cervical tuberculous lymphadenitis: diagnosis and demographics, a five-year case series in the UK. Ann R Coll Surg Engl . 2018;100(5):392-6.

14. Mittal P, Handa U, Mohan H, Gupta V. Comparative evaluation of fine needle aspiration cytology, culture, and PCR in diagnosis of tuberculous lymphadenitis. Diagn Cytopathol . 2011;39:822-6.

15. Derese Y, Hailu E, Assefa T, Bekele Y, Mihret A, Aseffa A, et al. Comparison of PCR with standard culture of fine needle aspiration samples in the diagnosis of tuberculosis lymphadenitis. J Infect Dev Ctries . 2012;6:53-7.

16. Omura S, Nakaya M, Mori A, Oka M, Ito A, Kida W, Inayoshi Y, Inoue A, Fuchigami T, Takamori $\mathrm{M}$, et al. A clinical review of 38 cases of cervical tuberculous lymphadenitis in Japan-The role of neck dissection. Auris Nasus Larynx . 2016;43(6):672-6.

17. Das S, Das D, Bhuyan UT, Saikia N. Head and neck tuberculosis: scenario in a tertiary care hospital of North Eastern India. Journal of clinical and diagnostic research: J Clin Diagn Res . 2016;10(1):MC04.

18. Marais BJ, Wright CA, Schaaf HS, Gie RP, Hesseling AC, Enarson DA, Beyers N, et al. Tuberculous lymphadenitis as a cause of persistent cervical lymphadenopathy in children from a tuberculosisendemic area.Pediatr Infect Dis J . 2006;25(2):142-6.

19. Katila ML, Brander E, Backman A. Neonatal BCG vaccination and mycobacterial cervical adenitis in childhood. Tubercle . 1987;68(4):291-6.

20. Ogundipe T, Otolorin A, Ogundipe F, Sran G, Abbas G, Filani O, et al. Multidrug-resistant tuberculosis lymphadenitis as the initial presentation of secondary multidrug-resistant tuberculosis: a case report. Cureus . 2018;10(3).

21. Datta S, Bhattacherjee S. Primary multi drug resistant extra-pulmonary tuberculosis presenting as cervical lymphadenitis. J Glob Infect Dis . 2014;6(2):91.

22. Mirsaeidi SM, Tabarsi P, Edrissian MO, Amiri M, Farnia P, Mansouri SD, Masjedi MR, Velayati AA, et al. Primary multi-drug resistant tuberculosis presented as lymphadenitis in a patient without HIV infection. Monaldi Arch Chest Dis . 2004;61(4).

23. Kant S, Saheer S, Hassan G, Parengal J. Extra-pulmonary primary multidrug-resistant tubercular lymphadenitis in an HIV negative patient. BMJ Case Rep . 2012;2012. pii: bcr0620114337.

24. Mittal N, Bansal P. Multidrug resistant extrapulmonary tuberculosis - three case reports and review of literature. Intern Med Inside . 2014;2:2.

25. Pablos-Méndez A, Raviglione MC, Laszlo A. Global surveillance for antituberculosis-drug resistance, 1994-1997. World Health OrganizationInternational Union against Tuberculosis and Lung Disease Working Group on Anti-Tuberculosis Drug Resistance Surveillance. N Engl J Med . 1998; 338:1641-9.

26. Nasiri MJ, Rezaei F, Zamani S, Darban-Sarokhalil D, Fooladi AA, Shojaei H, Feizabadi MM, et al. Drug resistance pattern of Mycobacterium tuberculosis isolates from patients of five provinces of Iran. Asian Pac J Trop Med . 2014;7(3):193-6.

27. Bahrmand AR, Titov LP, Tasbiti AH, Yari S, Graviss EA. High-level rifampin resistance correlates with multiple mutations in the rpoB gene of pulmonary tuberculosis isolates from the Afghanistan border of Iran. J Clin Microbiol . 2009;47(9):2744-50.

28. Shamaei M, Marjani M, Chitsaz E, Kazempour M, Esmaeili M, Farnia P, Tabarsi P, Amiri MV, Mirsaeidi M, Mansouri D, Masjedi MR, et al. First-line anti-tuberculosis drug resistance patterns 
and trends at the national TB referral center in Iran - eight years of surveillance.Int J Infect Dis . 2009;13(5):e236-40.

29. Brown HM, Abbitt PL, Wilkinson EJ. Diagnosis of clinically unsuspected extrapulmonary tuberculosis by fine needle aspiration: a case report.Acta Cytologica . 2001;45(6):1032-6.

30. Ormerod LP. Multidrug-resistant tuberculosis (MDR-TB): epidemiology, prevention and treatment. Br Med Bull . 2005;73(1):17-24.

31. harma SK, Mohan A, Gupta R, Kumar A, Gupta AK, Singhal VK and Pande JN, et al. Clinical presentation of tuberculosis in patients with AIDS: An Indian experience. Indian J Chest Dis Allied Sci.1997;39:213-20.

32. Tarashi S, Fateh A, Jamnani FR, Siadat SD, Vaziri F. Prevalence of Beijing and Haarlem genotypes among multidrug-resistant Mycobacterium tuberculosis in Iran: systematic review and metaanalysis. Tuberculosis . 2017;107:31-7.

33. Farnia P, Masjedi MR, Mirsaeidi M, Mohammadi F, Vincent V, Bahadori M, Velayati AA, et al. Prevalence of Haarlem I and Beijing types of Mycobacterium tuberculosis strains in Iranian and Afghan MDR-TB patients. J Infect . 2006;53(5):331-6.

34. Newberry DM, Bell TR. Congenital Tuberculosis: A New Concern in the Neonatal Intensive Care Unit. Adv Neonat Care . 2018;18(5):341-9.

35. Hamadeh MA, Glassroth J. Tuberculosis and pregnancy. Chest . 1992;101(4):1114-20.

36. Ormerod P. Tuberculosis in pregnancy and the puerperium. Thorax . 2001;56(6):494-9.

37. Cantwell MF, Shehab ZM, Costello AM, Sands L, Green WF, Ewing Jr EP, Valway SE, Onorato IM, et al. Congenital tuberculosis. New Engl J Med . 1994;330(15):1051-4.

38. Li RL, Wang JL, Wang XF, Wang MS. Tuberculosis in infants: a retrospective study in China. SpringerPlus . 2016;5(1):546.

39. Shah I, Poojari V, Meshram H. Multi-Drug Resistant and Extensively-Drug Resistant Tuberculosis. Indian J Pediatr. 2020 Feb 26:1-7.

40. Khazaei S, Rezaeian S. Challenges of achieving tuberculosis elimination by 2050: A need for more attention in the TB control program in Iran. Iran J Public Health . 2017;46(5):717-8.

41. Elder NC. Extrapulmonary tuberculosis: a review. Arch Fam Med . 1992;1(1):91.

42. Mittal N, Bansal P. Multidrug resistant extrapulmonary tuberculosis - three case reports and review of literature. Intern Med Inside . 2014;2:2.

43. Lee KC, Tami TA, Lalwani AK, Schecter G. Contemporary management of cervical tuberculosis. Laryngoscope . 1992;102(1):60-4.

44. Deveci HS, Kule M, Kule ZA, Habesoglu TE. Diagnostic challenges in cervical tuberculous lymphadenitis: A review. North Clin Istanb . 2016;3(2):150.

45. Azarkar Z, Arbabi A. Comparison of PPD test in household contacts of smear-positive and-negative tuberculosis (TB). Int J Mycobacteriol . 2015;4:95.

46. Gupta SK, Chugh TD, Sheikh ZA, al-Rubah NA. Cytodiagnosis of tuberculous lymphadenitis. A correlative study with microbiologic examination. Acta Cytol . 1993;37:329-32.

47. Dayal R, Agarwal D, Pathak H, Feroz S, Kumar M, Chauhan DS, Bhatia R, et al. PCR targeting IS6110 in diagnosing tuberculosis in children in comparison to MGIT culture. Indian J Tuberc . 2016;63(3):1547 .

48. Baek CH, Kim SI, Ko YH, Chu KC. Polymerase chain reaction detection of Mycobacterium tuberculosis from fine-needle aspirate for the diagnosis of cervical tuberculous lymphadenitis. Laryngoscope . 2000;110:30-4.

49. Chiesa Estomba CM, Betances Reinoso FA, Rivera Schmitz T, Ossa Echeverri CC, González Cortés MJ, Santidrian Hidalgo C, et al. Head and neck tuberculosis: 6-year retrospective study. Acta Otorrinolaringol Esp. 2016;67:9-14.

\section{Figure legend}

Figure 1. The CXR image of the patient revealed normal scene without remarkable sign except minor nodular and infiltrative lesion in the left lung. 


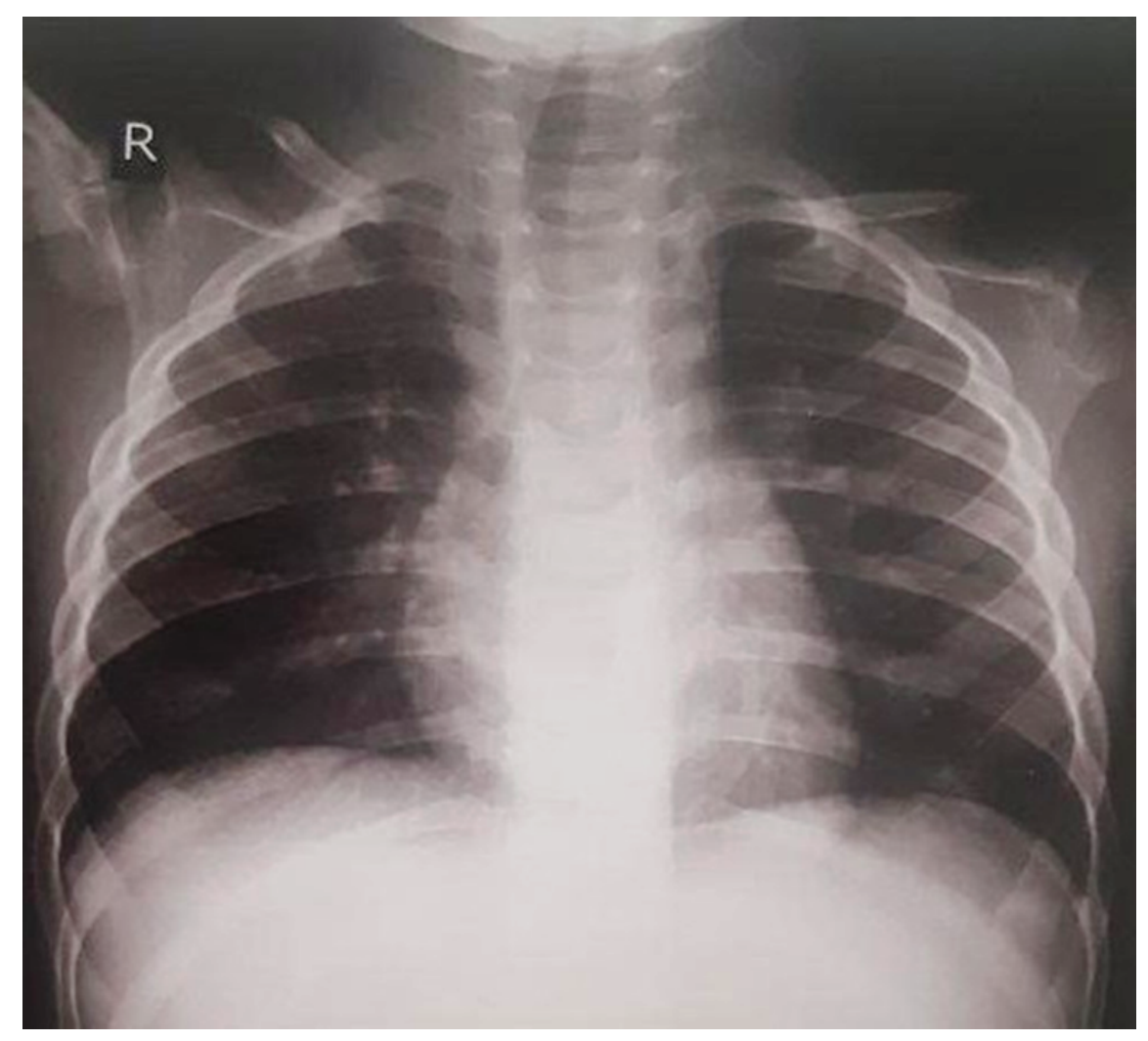

